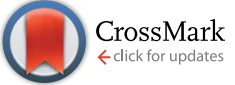

Cite this: RSC Adv., 2017, 7, 15500

\title{
Superparamagnetic iron oxide nanoparticles functionalized by peptide nucleic acids $\uparrow$
}

\author{
Marco Galli, ${ }^{a}$ Andrea Guerrini, ${ }^{b}$ Silvia Cauteruccio, ${ }^{a}$ Pramod Thakare, ${ }^{a}$ Davide Dova, ${ }^{a}$ \\ Francesco Orsini, ${ }^{\mathrm{C}}$ Paolo Arosio, ${ }^{\mathrm{C}}$ Claudio Carrara, ${ }^{a}$ Claudio Sangregorio, de \\ Alessandro Lascialfari, ${ }^{\text {ce }}$ Daniela Maggioni ${ }^{\star a e}$ and Emanuela Licandro ${ }^{\star a e}$
}

A novel efficient method has been developed for covalently linking Peptide Nucleic Acid (PNA) oligomers and superparamagnetic iron oxide nanoparticles (SPION), to produce water soluble hybrid nanomaterials that can act as MRI contrast agents, as hyperthermia promoters and as PNA carriers. The multistep procedure involves: (i) preparation of oleate-stabilized SPION by using the thermal decomposition method, to control the size of the magnetic core (here $17 \pm 2 \mathrm{~nm}$, by TEM measurement); (ii) exchange of the oleate layer by dimercaptosuccinic acid (DMSA), to impart water solubility and to provide functional groups for PNA grafting; (iii) functionalization of a PNA oligomer with a terminal maleimide moiety, to allow SPION-PNA conjugation by thiol-maleimide Michael addition reaction, exploiting the SH groups of DMSA on the SPION surface. The method was tested using a model PNA decamer containing all four nucleobases (-CTAGATCACT-). SPION-PNA conjugation by SH addition was found more efficient than conjugation through amide bond between the $\mathrm{COOH}$ groups of DMSA and the terminal $\mathrm{NH}_{2}$ groups of PNA. Elemental analysis, UV-Vis and IR spectra, and $\zeta$-potential measures confirmed the PNA binding (a loading of ca. 400 PNA strands per SPION was estimated, molar ratio ca. 1:15 with respect to DMSA). A detailed characterization of the morphology, relaxivity and magnetic properties of the SPION used for PNA binding is reported, and compared to the one relative to the SPION-PNA conjugate. The analysis of the magnetic behaviour showed that the nanoparticles are in the superparamagnetic regime at room temperature, and have a considerably high saturation magnetization $\left(85 \mathrm{emu} \mathrm{g}^{-1}\right.$ ). Magnetic hyperthermia measurements gave a remarkable value of the heat released (SAR $=65 \mathrm{~W} \mathrm{~g}^{-1}$ ), which makes these SPION suitable for magnetic hyperthermia treatments. The SPION were also able to effectively shorten both longitudinal and transverse relaxation times of water, with $r_{1}$ and $r_{2}$ values higher with respect to routinely used commercial contrast agents, at the typical fields of clinical instrumentation.

Received 12th January 2017 Accepted 3rd March 2017

DOI: 10.1039/c7ra00519a

rsc.li/rsc-advances

\section{Introduction}

The high current interest in the uses of multifunctional superparamagnetic iron oxide nanoparticles (SPION) in nanomedicine $^{\mathbf{1}}$ derives from their sensing, moving and heating abilities, imparted by their unique magnetic properties. The most common application is the use as contrast agents for magnetic resonance imaging (MRI). ${ }^{1 b, 2}$ In addition, in the presence of SPION, by means of an external ac magnetic field it

${ }^{a}$ Dipartimento di Chimica, Università degli Studi di Milano, Via Golgi 19, 20133 Milano, Italy.E-mail: daniela.maggioni@unimi.it

${ }^{b}$ LA.M.M. c/o Dipartimento di Chimica, Università degli Studi di Firenze, Via della Lastruccia 3, 50019 Sesto F.no (FI), Italy

${ }^{c}$ Dipartimento di Fisica, Università degli Studi di Milano, Via Celoria 20, 20133 Milano, Italy

${ }^{d}$ ICCOM - C.N.R., via Madonna del Piano 10, 50019 Sesto F.no (FI), Italy

${ }^{e}$ Consorzio INSTM, via G. Giusti 9, 50121, Firenze, Italy

$\dagger$ Electronic supplementary information (ESI) available. See DOI: 10.1039/c7ra00519a is also possible to induce local release of heat to the surrounding tissues, which can be exploited as a cancer therapy treatment called Magnetic Fluid Hyperthermia (MFH). ${ }^{3}$ Currently, MFH is under clinical testing associated with radiotherapy for the treatment of several tumours. ${ }^{4}$ Other important applications consist in cell labelling, separation and tracking ${ }^{5}$ and delivery of drugs and genes. ${ }^{6}$ The last application is particularly useful for preserving labile therapeutic species or for improving their bioavailability.

Peptide nucleic acids (PNAs) ${ }^{7}$ are synthetic polyamide mimics of natural DNA and RNA, in which the (desoxy)ribosephosphate backbone is replaced by $N$-(2-aminoethyl)glycyl neutral repeating units (Chart 1). The nucleobases, linked to the backbone through methylencarbonyl linkers, lie at the right distance to pair with the nucleobases of natural DNA or RNA single strand, with high affinity and sequence specificity.

The major advantages of PNAs are the resistance to enzymatic biodegradation by proteases and nucleases, and the higher chemical/thermal stability over their duplexes with DNA 


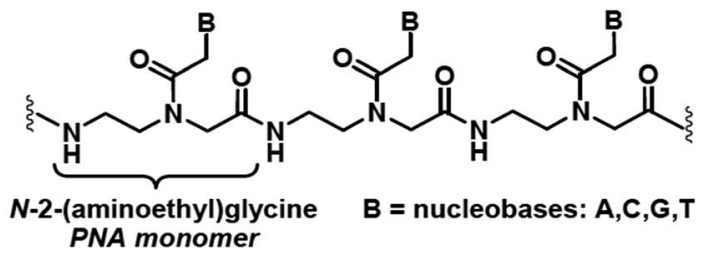

Chart 1 A peptide nucleic acid repeating fragment.

or RNA strands with respect to dsDNA and dsRNA. These features make PNAs an attractive tool for gene therapy, where the poor in vivo stability of nucleic acids and their ineffective uptake by target cells hamper translation to the clinic of effective platforms developed in in vitro cell assays. To pursue this end, however, it is necessary to enhance PNA bioavailability by conjugation to additional agents. Indeed, PNA major drawbacks are poor solubility in physiological media and very low ability to pass the cellular membranes.

The conjugation of PNA strands to superparamagnetic iron oxide nanoparticles could address these drawbacks, improving for example PNA solubility and cell permeability and, in a more general sense, improving its potential in biology and medicine. In fact, the system SPION-PNA can be suitable for gene analysis in diagnostics and gene therapy by taking advantage, on one hand, of ability of PNA to target in a very specific manner DNA and RNA sequences, and on the other hand by exploiting the superparamagnetic properties of nanoparticles, whose potential in biomedicine is well established. Very few studies appeared in the literature on the binding of PNA to magnetic iron oxide nanoparticles. ${ }^{\mathbf{8 9}}$ In the most recent report 9 the covalent binding was obtained by a multistep procedure, involving functionalization of the surface of naked magnetite nanoparticles with 3-aminopropyltriethoxysilane, followed by reaction with acryloyl chloride, to give acrylamide-grafted SPION, radical polymerization and further covalent functionalization of the polymer coating with streptavidin. Pyrrolidinyl PNA previously functionalized with biotin was finally conjugated to the nanoparticles, via specific biotin-streptavidin interaction. A versatile and effective synthetic platform for grafting PNA oligomers to naked commercial maghemite NPs had been previously developed, ${ }^{8}$ based on the high affinity of siloxane group for iron oxide. However, the solubility of the PNA-based hybrid nanostructures obtained by this method was not satisfactory. ${ }^{8}$ Here we describe a novel synthetic strategy, which gives access to hybrid inorganic-organic systems made of iron oxide nanoparticles and PNA strands, for applications as contrast agents for MRI and heat mediators in MFH. The method here reported combines easiness and efficacy with the possibility to tune the SPION size, and allows the obtainment of magnetic PNA with good water solubility.

\section{Results and discussion}

\subsection{Synthesis of the iron oxide nanoparticles}

The synthesis of highly monodisperse magnetic iron oxide nanoparticles was extensively addressed in the last years by many research teams. The discovery of thermal decomposition methods enabled a dramatic increase of monodispersion, ${ }^{\mathbf{1 0}}$ but reproducibility issues are not yet entirely overcome. Actually, many factors affect the size and the crystallinity, and then the magnetic properties of the sample, such as the precursor nature, the heating ramp, the aging time and temperature, the solvent, the molar ratio between the iron precursor and the surfactant.

Here we adopted a synthetic methodology in which the iron(0) precursor $\mathrm{Fe}(\mathrm{CO})_{5}$ is decomposed in high-boiling 1octadecene, in the presence of oleic acid (OA) as capping agent. ${ }^{11}$ The literature procedure used a $4: 1 \mathrm{OA}:$ Fe molar ratio that gave spherical SPION with an average diameter of $10 \mathrm{~nm}$. It is known that the SPION size can be tuned by varying the relative concentrations of iron precursor and capping agent. ${ }^{\mathbf{1 2 , 1 3}}$ In line with this, on increasing the OA : Fe ratio (see Table 1) we found a concomitant increase both of the hydrodynamic diameter (measured by dynamic light scattering analyses, DLS, Fig. S1 ESI $\dagger$ ) and of the diameter measured by transmission electron microscopy (TEM, Fig. S2 in ESI $\dagger$ ). The size increment was dramatically sensitive to $\mathrm{OA}$ : Fe ratio, as can be clearly observed in the graphs reported in Fig. S3 (ESI $\dagger$ ). As expected, the mean particle size distributions measured by DLS were significantly larger than the ones obtained by TEM measurements, since DLS returns a hydrodynamic diameter that takes into account not only the magnetic core, but also the OA shell, and the corona shell of solvent interacting with OA, that jointly fluctuates by Brownian motions.

The SPION7 sample, obtained with an OA : Fe ratio equal to 7 , consisted in highly spherical monodisperse nanoparticles with a mean diameter of $17.0 \mathrm{~nm}$. This sample was chosen for carrying on the work, because its size grants for the highest hyperthermic efficiency among the series of samples. Moreover, the other two samples exhibited several shortcomings (polydispersion, polycrystalline/amorphous structure), which are detrimental for their magnetic properties. Furthermore, the size of SPION7 allows reaching stability once dispersed in water (see below), avoiding possible aggregation favoured by the high magnetic moment of bigger nanoparticles, the last effect highly undesirable for biomedical applications. Therefore, the core of all the nanosystems described hereafter was constituted by the SPION7 sample, even if not explicitly stated.

Table 1 Mean size of the SPION obtained, in ESI with different OA : Fe molar ratios, as measured by DLS (hydrodynamic diameters, intensities distribution) and by TEM

\begin{tabular}{llcl}
\hline Sample & $\begin{array}{l}\text { OA }: \text { Fe } \\
\text { molar ratio }\end{array}$ & DLS (intensities) $(\mathrm{nm})$ & TEM $(\mathrm{nm})$ \\
\hline SPION6 & $6: 1$ & $23.3 \pm 1.6^{a}$ & $11.9 \pm 3.0$ \\
SPION7 & $7: 1$ & $26.6 \pm 3.0$ & $17.0 \pm 1.9$ \\
SPION8 & $8: 1$ & $38.95 \pm 9.2$ & $29.9 \pm 2.6^{b}$
\end{tabular}

${ }^{a}$ The population with a larger size $(78 \mathrm{~nm})$ observable in Fig. S1 (ESI) represented only a very minor fraction of the whole sample $(1.5 \%$, according to the DLS number distribution). ${ }^{b} \mathrm{~A}$ population with a smaller size $(13.5 \pm 3.4 \mathrm{~nm})$ was also observed by TEM, probably due to uncompleted Ostwald ripening (Fig. S2, ESI). 


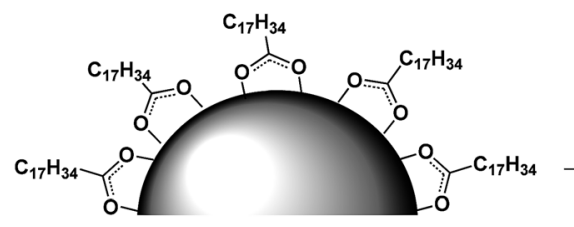

SPION@OA

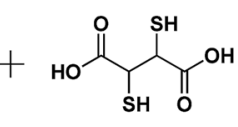

DMSA

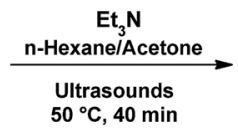

$50{ }^{\circ} \mathrm{C}, 40 \mathrm{~min}$

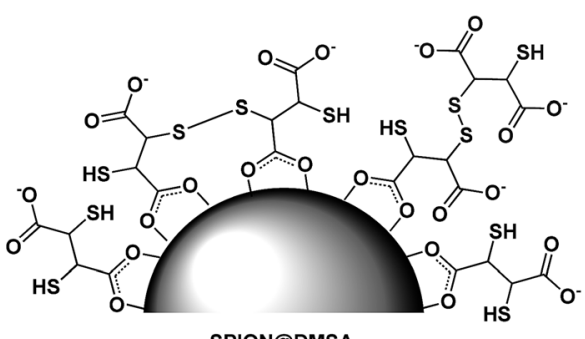

SPION@DMSA

Scheme 1 The ligand exchange procedure affording the SPION@DMSA here used for PNA conjugation. Different binding modes of the carboxylate groups on the magnetite surface are depicted in the scheme, ${ }^{16}$ taking into account also the possibility of oxidative coupling of SH groups. ${ }^{17}$

In order to impart the necessary water solubility and biocompatibility, the oleate layer was exchanged with dimercaptosuccinic acid (DMSA). Actually nanoparticles coated by DMSA are stabilized over a wide $\mathrm{pH}$ range by the electrostatic repulsion arising from the negative charge present on their surface even at relatively low $\mathrm{pH}$ values ( $\mathrm{p} K_{\mathrm{a}}$ of DMSA: $2.71,3.43$, $9.65,12.05) \cdot{ }^{14}$ Besides providing SPION stability in physiological conditions, DMSA offers the further benefit of two functional groups ( $\mathrm{COOH}$ and $\mathrm{SH})$, which both can be exploited for the covalent bonding of a variety of organic molecules. ${ }^{15}$

To perform the ligand exchange we used a literature methodology $^{16}$ (see Scheme 1), modified by the use of sonication during the reaction, which allowed a significant shortening of the exchange time ( $40 \mathrm{~min} v s .12-24 \mathrm{~h}$ of the literature procedures), while maintaining the quasi-quantitative recovery of the water-dispersed SPION.

The so obtained SPION@DMSA suspensions, stocked at $4{ }^{\circ} \mathrm{C}$ under $\mathrm{N}_{2}$ at $\mathrm{pH}>9$, were very stable for several months. The $\zeta-$ potential (Fig. S4, ESI $\dagger$ ) was negative even at quite low pH, as expected from the above reported $\mathrm{p} K_{\mathrm{a}}$ values. DLS measurements performed on samples at pH 9 showed (Fig. S5, ESI $\dagger$ ) in the distribution size graph (intensities) two distinct peaks centred at $146 \pm 42 \mathrm{~nm}$ and $44 \pm 8 \mathrm{~nm}$, indicating a certain degree of aggregation. However, the hydrodynamic distribution by numbers showed that the aggregated population represented only a minority of the whole sample of suspended particles $(2.5 \%)$.

The morphology of DMSA-stabilized SPION was investigated by Tapping Mode Atomic Force Microscopy (TM-AFM), which showed that the nanoparticles maintained the spherical-like shape observed before ligand exchange (see Fig. 1a). By analysis of several images, the overall size (magnetic core and DMSA shell coating) was estimated as $20.6 \pm 2.0 \mathrm{~nm}$ (averaged value over 40 observations), in the same range as observed by TEM for precursorSPION@OA, and significantly smaller than the value measured by DLS for SPION@DMSA, due to the contribution to the hydrodynamic diameter of the solvating water that surrounds the NPs.

AFM phase images have also been acquired, to evidence the coating shell surrounding the iron core. Such images are produced by mapping the phase angle shifts across the
$\mathrm{H}_{3} \stackrel{+}{\mathrm{N}}-\mathrm{GTAGATCACT}-\mathrm{CONH}_{2}$ 1

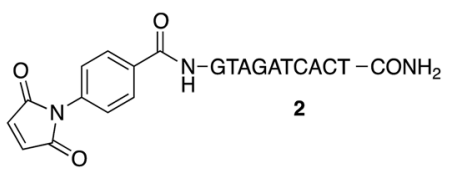

Chart 2 The two PNA decamers here prepared.
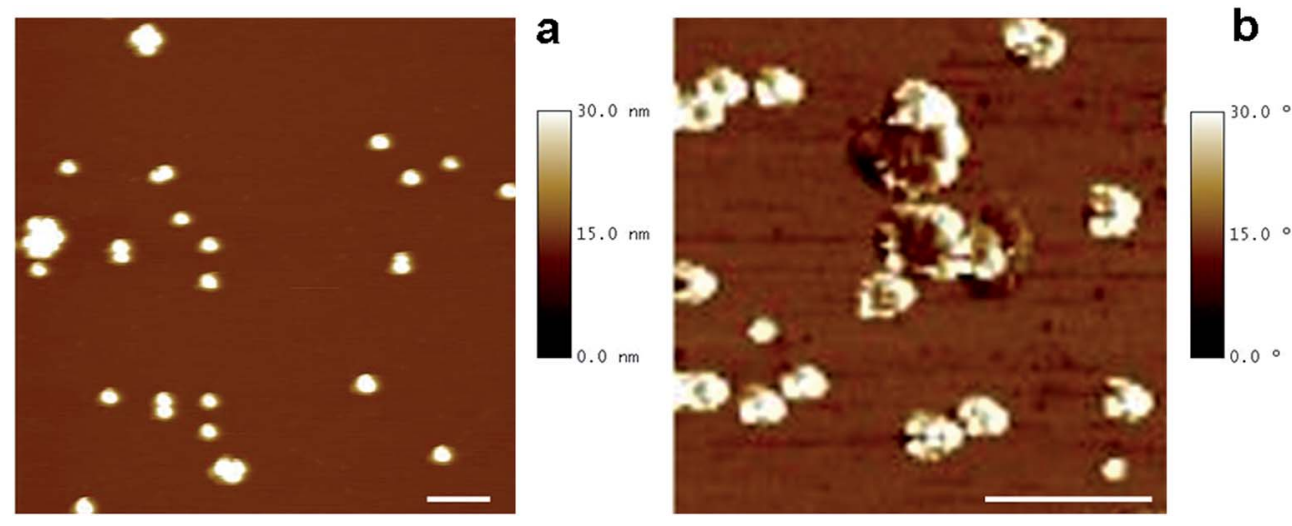

Fig. 1 AFM images of SPIONADMSA samples adsorbed on a mica support. (a) AFM topography image. Besides single SPION, some small aggregates are also observed, most likely formed in the deposition step. Scan area $800 \times 800 \mathrm{~nm}^{2}$. Vertical scale $30 \mathrm{~nm}$. (b) AFM phase image, which allows the visualization of the coating (white rings) and of the NP magnetic core (darker areas, partially eclipsed by the external coating). Scan area $300 \times 300 \mathrm{~nm}^{2}$. For both the images, scale bar $=100 \mathrm{~nm}$. 

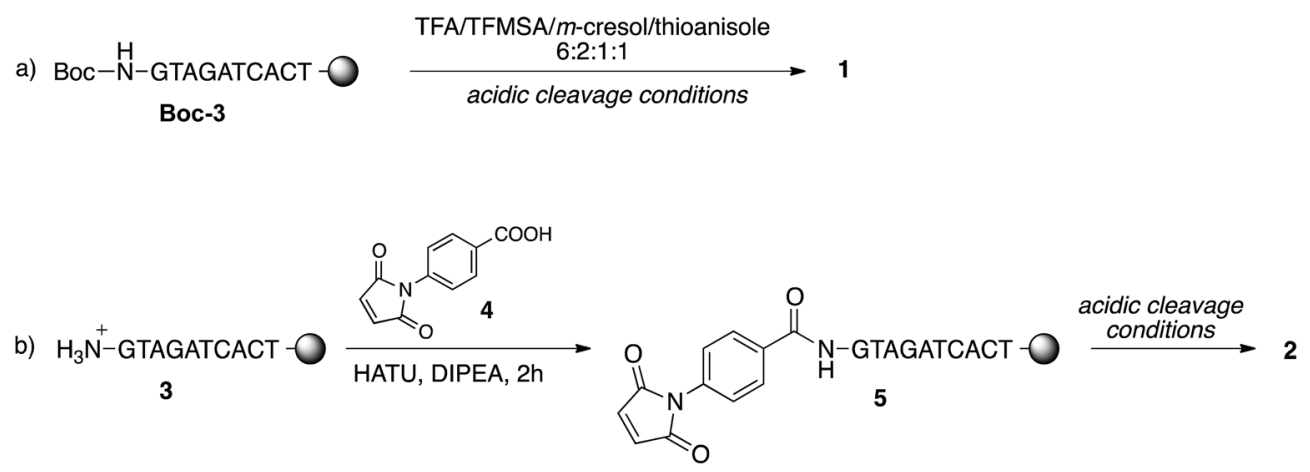

Scheme 2 The synthesis of PNA decamers 1 and 2 from the resin-supported PNA decamers Boc-3 and 3.

sample ${ }^{18}$ which bring information about mechanical properties besides the topography features. As shown in Fig. 1b, AFM phase image allows the visualization of the soft NP coating (white areas) as well as of their stiffer magnetic core (darker areas).

\subsection{Preparation of PNA decamers 1 and 2}

To study the conjugation of the PNA strand onto SPION@DMSA, we selected, as model, a PNA decamer sequence containing all four nucleobases, namely GTAGATCACT. Since DMSA contains two different functional groups $(-\mathrm{COOH}$ and - $\mathrm{SH}$ ), we synthesized the two PNA sequences 1 and 2 (Chart 2), displaying a terminal free amine group and a maleimido group, respectively, to verify the possibility of conjugation of PNA onto SPION through the amide bond formation reaction between PNA 1 and -COOH groups of DMSA, or through a Michael-type addition between PNA 2 and -SH groups of DMSA.

For this purpose, the resin-supported PNA decamer 3 (Scheme 2) was prepared by an automated solid-phase synthesis, using standard Boc/Z chemistry (see Experimental). The corresponding PNA decamer 1 was then obtained through the acidic treatment of Boc-3 (Scheme 2), which enables the cleavage of PNA 1 from the resin, removing at the same time all the Boc/Z protecting groups, followed by RP-HPLC purification of crude 1 (see Experimental). The preparation of PNA 2 was accomplished through the introduction of the maleimido moiety using $p$-maleimidobenzoic acid (4), ${ }^{19}$ which was manually coupled to the terminal $\mathrm{NH}_{2}$ group of the resin-supported PNA 3 by forming the amide bond with HATU as the condensing agent (eqn (b) in Scheme 2).

Thanks to the stability of the maleimido group toward the strong acidic conditions used for the cleavage of PNA from the resin, PNA 2 could be easily obtained from resin $\mathbf{5}$, and purified by RP-HPLC. The purity and identity of PNA 1 and 2 were confirmed by HPLC and MALDI-TOF analyses.

\subsection{Conjugation of PNA to SPION}

For the conjugation of the PNA strand to SPION, the two methods depicted in Scheme 3 were investigated. The widely used $^{20}$ coupling reaction between $\mathrm{COOH}$ and $\mathrm{NH}_{2}$ groups, mediated by the zero-length cross linker EDC (1-ethyl-3-(3dimethylaminopropyl)carbodiimide hydrochloride), was performed in the presence of NHS ( $N$-hydroxysuccinimide), which gave the corresponding succinimidyl ester of the $\mathrm{COOH}$ groups, more stable in the aqueous environment then the corresponding isourea intermediate ester (bottom of Scheme 3). This link strategy has been previously used also for binding functional molecules (such as PEG, ${ }^{21}$ 2-deoxyDglucose, ${ }^{22}$ or the NIRF dye
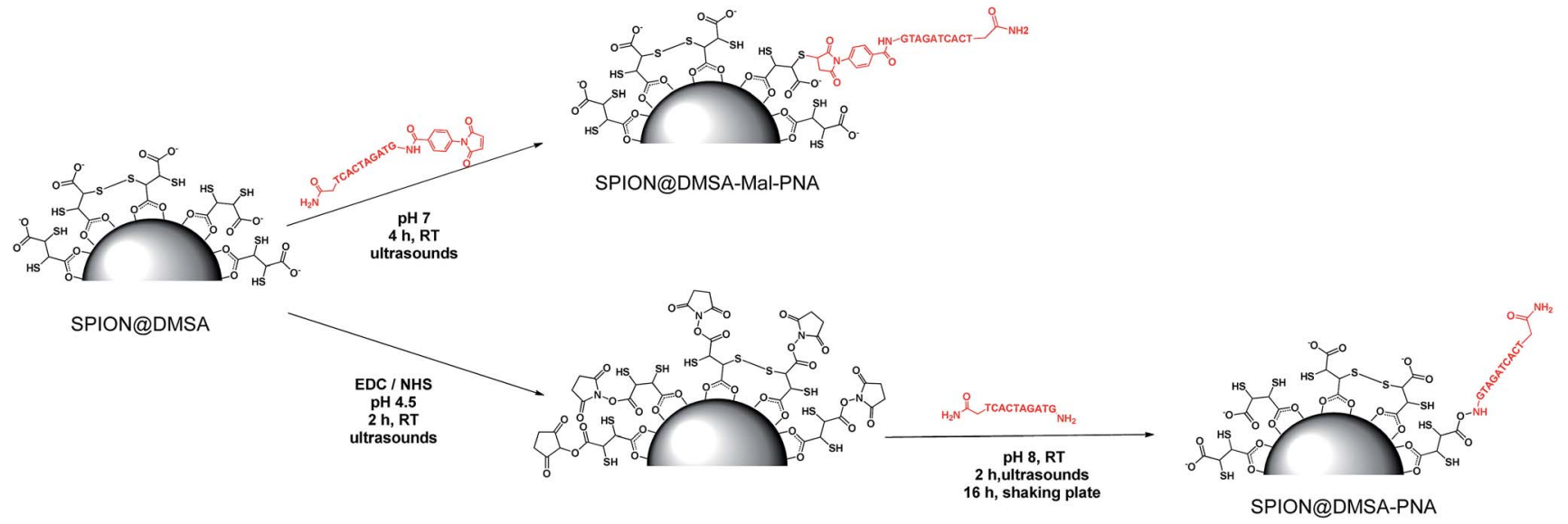

Scheme 3 Schematic representation of the two investigated routes for binding PNA to SPION@DMSA. 
Table 2 Elemental analysis of the SPION-PNA conjugates here prepared and of the precursors SPION@DMSA and PNA 1 and 2

\begin{tabular}{lcllr}
\hline & SPION@DMSA-Mal-PNA & SPION@DMSA-PNA & SPION@DMSA & PNA 1 $^{b}$ \\
\hline C\% & 10.23 & 8.03 & 3.36 & 47.57 \\
H\% & 1.40 & 1.73 & 0.75 & 5.03 \\
N\% & 3.97 & 2.16 & n.d. & 48.85 \\
C/N ratio & $1.73^{c}$ & $2.16^{c}$ & - & 29.79 \\
\end{tabular}

${ }^{a}$ Nanoparticles prepared by the amide bond formation between the $\mathrm{NH}_{2}$ of PNA and the COOH groups on SPION (see Scheme 3 for clarity). ${ }^{b}$ Calculated values. ${ }^{c}$ Calculated by subtracting the C\% due to DMSA from the total C\%.

IRDye800CW ${ }^{23}$ ) to the surface of NPs covered with DMSA. Unexpectedly, in our case this method did not work well. Some irreversible aggregation was observed (even in repeated trials) just a few minutes after the addition of $\mathrm{EDC} \cdot \mathrm{HCl}$ to the initially clear suspension of SPION@DMSA and NHS, which likely hampered the subsequent conjugation with PNA 1. In fact, the elemental analysis of the isolated final product clearly indicated that PNA was not the main species linked to the SPION surface, the measured $\mathrm{C} / \mathrm{N}$ ratio being much higher (2.16, after subtraction of the carbon content due to DMSA, see Table 2) than expected for bound PNA (1.60).

A different approach was therefore developed, involving the Michael addition of a thiol group of DMSA to the double bond of the maleimide-functionalized PNA 2 (top of Scheme 3). An Ellman's assay was preliminarily performed, to assure the presence of accessible SH groups on the SPION surface (see ESI†).

Moreover, before moving to the study of the conjugation of PNA 2 onto SPION@DMSA, the feasibility of the addition of SPION surface SH groups to a maleimide moiety was tested using a maleimide-rhodamine B adduct, expressly prepared by reacting 2-(aminoethyl)-maleimide with rhodamine-B isothiocyanate, as depicted in Scheme S1 (ESI $\dagger$ ) and detailed in the Experimental. $^{24}$ The reaction between the rhodaminemaleimide adduct and SPION@DMSA was conducted for $24 \mathrm{~h}$ at room temperature in the dark, to avoid the early

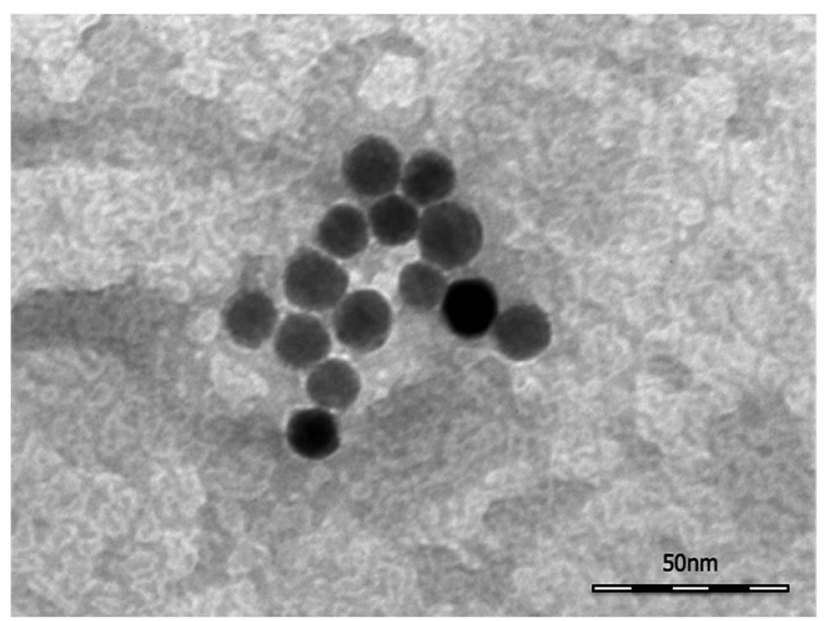

Fig. 2 A representative bright field TEM image of SPION@DMSA-MalPNA. photochemical decomposition of the chromophore. After accurate washing of the SPION (see Experimental), to remove all the unreacted chromophores, an UV-Vis spectrum of the suspension showed the superposition of the typical rhodamine peaks at 528 and $559 \mathrm{~nm}$ with the broad absorption/scattering profile of iron oxide SPION (Fig. S6, ESI†े). The UV-Vis spectrum of the supernatant, measured after a further centrifugation cycle, showed very weak absorptions, due both to rhodamine and iron oxide SPION, attributable to the slight fraction of nanoparticles not removed in the centrifugation procedure. This indicated that the maleimide-rhodamine adduct had been really grafted to SPION, further confirming the presence on the nanoparticle surface of SH groups suitable for the Michael addition reaction.

On the basis of these results, the functionalized PNA 2 was reacted with SPION@DMSA, and the Michael addition was completed in $4 \mathrm{~h}$ at room temperature at neutral $\mathrm{pH}$, under ultrasonic irradiation. The colloidal suspension, which remained clear throughout the coupling procedure, was centrifuged to isolate the functionalized nanoparticles (SPION@DMSA-MalPNA), while removing the unreacted PNA excess.

\subsection{Characterization of SPION@DMSA-Mal-PNA}

TEM images (Fig. 2) collected on the SPION@DMSA-Mal-PNA demonstrated that, as expected, the conjugation process did

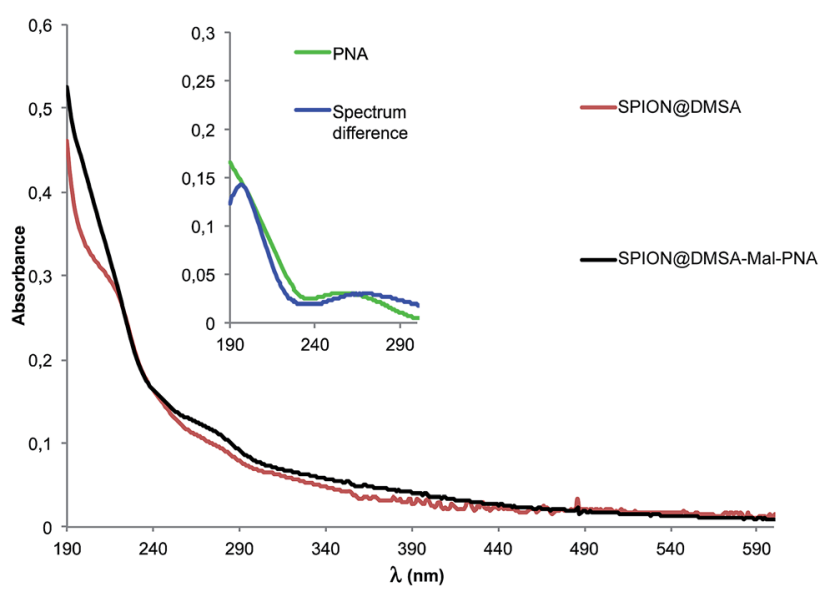

Fig. 3 UV-Vis spectra of water suspended SPION@DMSA-Mal-PNA (black trace) and SPION (DDMSA (red trace). Inset: blue trace, spectrum difference between SPIONADMSA-Mal-PNA and SPION@DMSA; green trace, spectrum of maleimide-PNA. 
a)

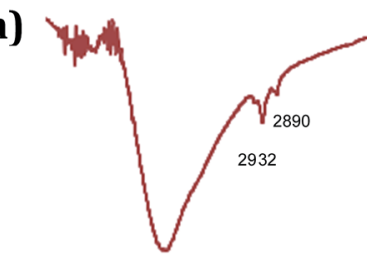

b)

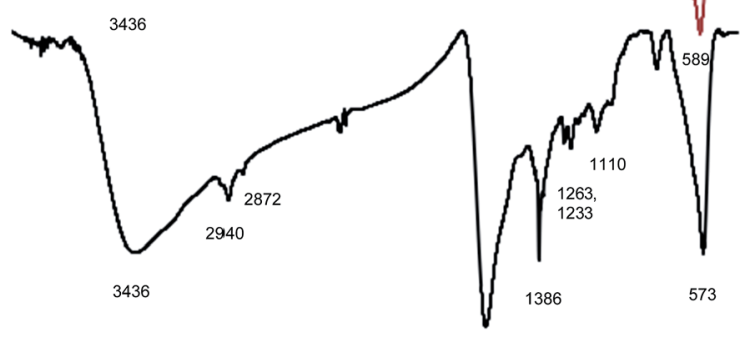

1658

\begin{tabular}{llllllll}
\hline & 1 & 1 & & \\
3900 & 3400 & 2900 & 2400 & 1900 & 1400 & 900 & 400
\end{tabular}

Wavelengt $h\left(\mathrm{~cm}^{-1}\right)$

Fig. 4 FTIR spectra (KBr pellet) of (a) SPION@DMSA; (b) SPION@DMSA-Mal-PNA.

not affect neither the morphology nor the average size of the NP magnetic cores.

Moreover, DLS measurements showed the same size distribution with respect to the unfunctionalized SPION@DMSA (Fig S5, ESI $\dagger$ ), proving that the conjugation of NPs to PNA did not affect the aggregation state.

The UV-Vis spectrum of SPION@DMSA-Mal-PNA suspended in water is reported in Fig. 3 together with that of the SPION@DMSA precursor. Their spectral difference (blue trace of the inset) clearly highlights the presence of the typical absorption peak of PNA nucleobases at $260 \mathrm{~nm}$, slightly shifted with respect to the position $(250 \mathrm{~nm})$ for the free maleimidePNA sequence (green trace in the inset), in line with previous evidence obtained on maghemite-PNA adducts. ${ }^{25}$

The adducts have also been investigated by FTIR spectroscopy and Fig. 4 compares the spectrum of SPION@DMSA-MalPNA (lower trace) with that of SPION@DMSA (upper trace). In the latter trace, the absorption at $589 \mathrm{~cm}^{-1}$ is attributable to the $\mathrm{Fe}-\mathrm{O}-\mathrm{Fe}$ stretching modes of the spinel structure of magnetite, ${ }^{17,26}$ while the presence of DMSA is indicated by the weak absorptions at about $2900 \mathrm{~cm}^{-1}$ (due to aliphatic $\nu_{\mathrm{CH}}$ modes), and by the broad bands at 1632 and $1396 \mathrm{~cm}^{-1}$, which are related to the asymmetric and symmetric stretching of the $\mathrm{COO}^{-}$groups of DMSA. ${ }^{27}$ The strong and broad $\nu_{\mathrm{OH}}$ band at $c a$. $3400 \mathrm{~cm}^{-1}$ indicates the presence of water on the nanoparticle surface, and therefore the absorption at $1632 \mathrm{~cm}^{-1}$ also includes contribution from the $\mathrm{OH}$ bending modes. The main difference between the two traces of Fig. 4 is constituted by the presence in the lower trace of a series of relatively weak absorptions in the $1100-1400 \mathrm{~cm}^{-1}$ region, due to the $\mathrm{C}-\mathrm{N}$ stretching modes of the nucleobases and of the PNA amide skeleton. ${ }^{28}$ The nucleobases and the amide CO groups of PNA also contribute to the broad absorption at $c a .1660 \mathrm{~cm}^{-1}$, which is more intense and slightly shifted with respect to the spectrum of the SPION@DMSA precursor.

To prove the effectiveness of the addition of PNA 2, elemental analyses were performed on the dried nanoparticles. Data are reported in Table 2.

The analyses showed the expected significant increase of the $\mathrm{C}$ and $\mathrm{N}$ content after interaction with PNA (the $\mathrm{H} \%$ values were not considered, since the use of water as solvent affects the reliability of these data). Noteworthy, the $\mathrm{C} / \mathrm{N}$ ratio for the SPION@DMSA-Mal-PNA adduct (after subtraction of the DMSA contribution) was in perfect accord with the calculated value for the Mal-PNA strand here used. On these bases, the PNA loading onto the SPION@Mal-PNA was calculated to be about 14\% w/w, corresponding to a molar ratio of $c a$. 1:15 with respect to DMSA. Each SPION@DMSA-Mal-PNA nanoparticle, which is covered by $c a$. 6000 DMSA molecules, is therefore loaded with ca. 400 PNA strands.

On the contrary, the $\mathrm{C} / \mathrm{N}$ ratio for the nanoparticles SPION@DMSA-PNA prepared from PNA 1 (Scheme 3) was much higher than the one of PNA, suggesting that, as above discussed, the sample was largely contaminated by impurities with a higher $\mathrm{C} / \mathrm{N}$ ratio, likely deriving from reagents (EDC, NHS) used in the coupling procedure and only partially removed.

Finally, $\zeta$-potential measurements on the SPION@DMSAMal-PNA colloid (Fig. 5) showed a decrease of the negative surface charge with respect to SPION@DMSA, from $-44.5 \pm$ $1.0 \mathrm{mV}$ to $-27.7 \pm 4.0 \mathrm{mV}$, in line with the bonding of a positively charged species such as PNA to the negatively charged native nanoparticles.

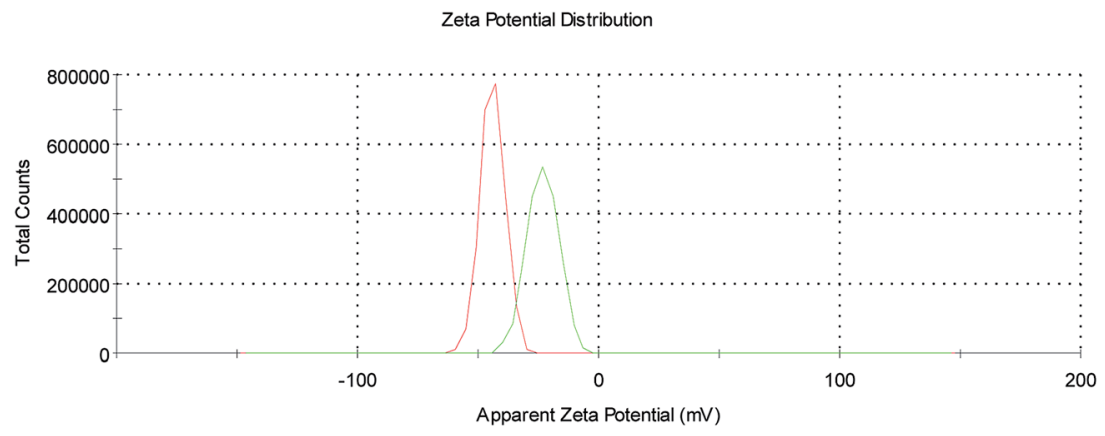

Fig. $5 \zeta$-Potential profiles of SPION@DMSA (red trace) and SPION@DMSA-Mal-PNA (green trace) in water, at pH 7.4. 
Table 3 Blocking temperature $\left(T_{\mathrm{B}}\right)$, coercive field $\left(H_{\mathrm{C}}\right)$, saturation magnetization $\left(M_{S}\right)$ and reduced remanent magnetization $\left(M_{0} T_{T} / M_{5}\right)$ of SPION7 nanoparticles with different coatings

\begin{tabular}{lllll}
\hline & $T_{\mathrm{B}}(\mathrm{K})$ & $H_{\mathrm{C}}(\mathrm{Oe})$ & $M_{\mathrm{S}}\left(\mathrm{emu} \mathrm{g}^{-1}\right)$ & $M_{0 \mathrm{~T}} / M_{5 \mathrm{~T}}$ \\
\hline SPION@OA & 250 & 460 & 87 & 0.30 \\
SPION@DMSA & 270 & 430 & 82 & 0.36 \\
SPION@DMSA-Mal-PNA & 270 & 430 & 80 & 0.36 \\
\hline
\end{tabular}

\subsection{Magnetic and relaxivity properties of SPION}

Thermal energy released by SPION during the action of an alternating magnetic field is the crucial parameter for the success of the MFH treatment. The capability of releasing heat is normally quantified by the SAR (Specific Absorption Rate), which describes the absorbed power per mass unit. In the linear regime, i.e. when the magnetization varies linearly with the magnetic field (low field amplitude and low energy barrier compared to thermal energy) the SAR depends on the field parameters and on the intrinsic properties of the SPION (eqn (1)), ${ }^{29 a}$ where $\nu$ and $H_{0}$ are the frequency and amplitude of the oscillating field, $M_{\mathrm{S}}$ is the saturation magnetization, $T$ the temperature, $k_{\mathrm{B}}$ the Boltzmann constant, $\rho$ the mass density, $V$ the particle volume and $\tau$ the time required by the particle's magnetic moment to reverse its orientation.

$$
\mathrm{SAR}=\frac{\pi \nu \mu_{0}^{2} H_{0}^{2} M_{\mathrm{S}}^{2} V}{3 \rho k_{\mathrm{B}} T} \frac{2 \pi \nu \tau}{1+(2 \pi \nu \tau)^{2}}
$$

Thus, to maximize the SAR, SPION must exhibit specific magnetic characteristics. ${ }^{29}$ Indeed, despite the SAR can be also increased by using high frequencies and high magnetic fields, these parameters cannot be increased above a fixed threshold, due to severe limitation imposed by the biological systems. ${ }^{30}$ On one hand, the SAR reaches its maximum value when the reversal time equals the time of the magnetization corresponding to the working frequency $\nu=183 \mathrm{kHz}$, i.e. $\tau=1 /(2 \pi \nu)$. For magnetite NPs this condition is satisfied for size in the $16-19 \mathrm{~nm}$ range $\mathrm{e}^{31}$ as is the case of SPION7. On the other hand, larger SAR will be obtained with higher saturation magnetization, which therefore should be as close as possible to the bulk magnetite value (92 $\left.\mathrm{emu}^{-1}\right)$. $^{32}$

Magnetic measurements were performed on powder samples of SPION7, (which is here indicated simply as SPION, as stated at point 2.1 above) both before and after the ligand exchange, using a SQUID magnetometer. The main magnetic parameters of the measured samples are listed in Table 3.

As shown in Fig. 6a the zero field cooled-field cooled (ZFCFC) magnetizations of SPION@OA display the characteristic blocking process of single domain NPs. The FC magnetizations exhibit a plateau at low temperature, which can be ascribed to interparticle interactions, which are not negligible when NPs are measured as dried powders. The blocking temperature, estimated as a first approximation from the maximum of the ZFC curve, is $T_{\mathrm{B}}=250 \mathrm{~K}$, indicating that at room temperature the NPs are in the superparamagnetic regime, which is a fundamental requisite for the proposed applications. This result is confirmed by the absence of remanence (Fig. 6b) observed in the room temperature magnetization $v s$. field curve. Conversely, at low temperature (Fig. 6c) magnetic irreversibility is observed, with a coercive field $H_{\mathrm{C}}=500$ Oe and reduced remanence $R=M_{\mathrm{R}} / M_{\mathrm{S}}=0.3$, comparable with the data reported in the literature for magnetite nanoparticles of similar size. ${ }^{33}$ Most importantly, the saturation magnetization is high $\left(M_{\mathrm{S}}=\right.$
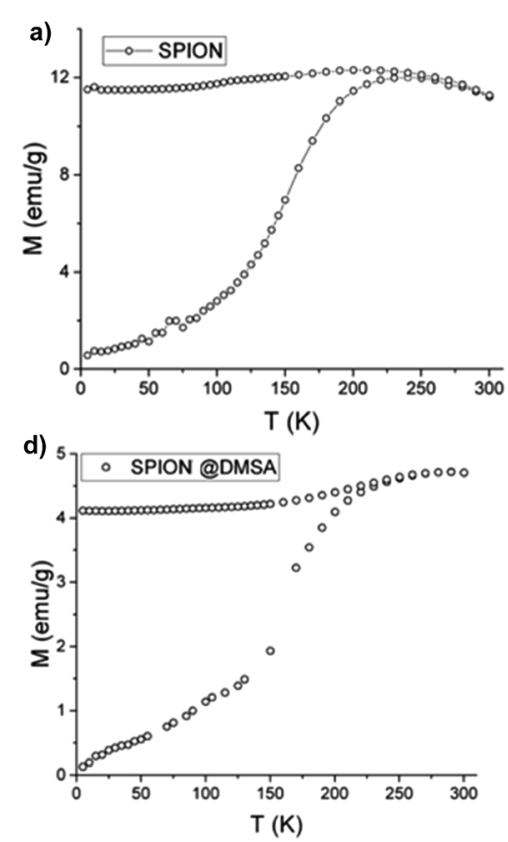

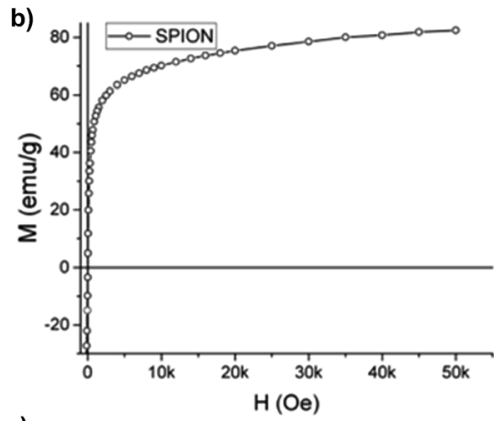

e)

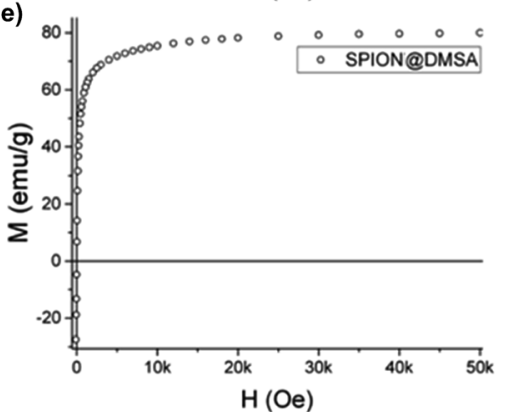

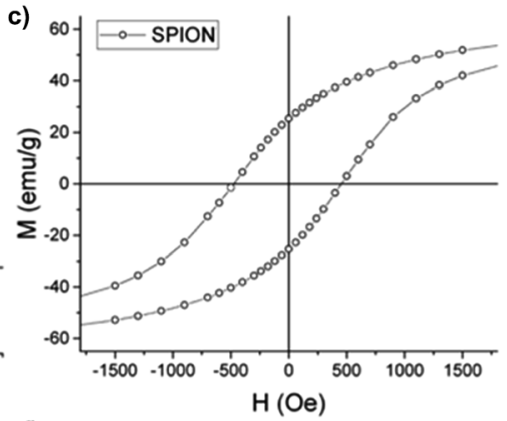

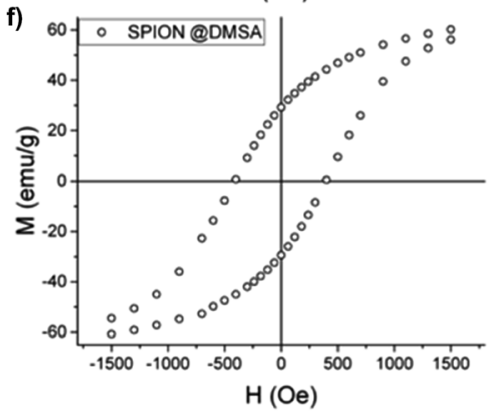

Fig. 6 Magnetic data on SPIONaOA (upper panels) and SPIONaDMSA (lower panels): (a and d) ZFC-FC magnetization curves; (b and e) magnetization at $300 \mathrm{~K}$; (c and f) enlargement of the low field region of the hysteresis cycle at $2.5 \mathrm{~K}$. 
$85 \mathrm{emu} \mathrm{g}^{-1}$ ) and comparable to the value of bulk magnetite. A fit to the Langevin function of the low field part of the $M(H)$ curve $^{34}$ gives a magnetic moment value per particle, $\mu$, of $c a .5 \times 10^{4} \mu_{\mathrm{B}}$, which is consistent with the size and magnetization of the NPs, confirming their good quality.

The magnetic data of SPION@DMSA reported in Fig. 6d-f show that the ligand exchange does not affect the good magnetic characteristics: $T_{\mathrm{B}}$ lower than room temperature, no remanence at $300 \mathrm{~K}$ and high $M_{\mathrm{S}}$ value. The same holds true for the NPs after their conjugation to PNA (Fig. S7, ESI $\dagger$ ).

Magnetic hyperthermia measurements were made by recording the evolution of temperature of the SPION@DMSA samples dispersed in water $\left(0.77 \mathrm{mg} \mathrm{mL}^{-1} \mathrm{Fe}\right)$ exposed to an alternating magnetic field of $17 \mathrm{kA} \mathrm{m}^{-1}$ and $183 \mathrm{kHz}$ for 5 minutes. The temperature kinetics is shown in Fig. 7. Despite the small temperature increase observed, the sample has a remarkable SAR $=65 \mathrm{~W} \mathrm{~g}^{-1} \mathrm{Fe}$, which makes it a suitable candidate for future application in MFH treatments.

The ability of SPION@DMSA to affect water nuclear relaxation times was also measured, to evaluate their effectiveness as MRI contrast agents. In Fig. 8 the ${ }^{1} \mathrm{H}$-NMR dispersion (NMRD) profiles for SPION@DMSA as well as for SPION@DMSA-MalPNA are reported, where the nuclear longitudinal $\left(r_{1}\right)$ and transverse $\left(r_{2}\right)$ relaxivities were evaluated in the usual way (eqn (2)), in which $\left(1 / T_{\mathrm{i}}\right)_{\text {meas }}$ indicates the measured value on the sample with iron concentration $c=0.5 \mathrm{mmol} \mathrm{L}^{-1}$, and $\left(1 / T_{\mathrm{i}}\right)_{\mathrm{dia}}$ the nuclear relaxation rate of the water used as host solution.

$$
r_{\mathrm{i}}=\left[\left(1 / T_{\mathrm{i}}\right)_{\mathrm{meas}}-\left(1 / T_{\mathrm{i}}\right)_{\mathrm{dia}}\right] / c, i=1,2
$$

It can be evinced that the sample is able to effectively shorten both longitudinal $\left(T_{1}\right)$ and transverse $\left(T_{2}\right)$ relaxation times, and therefore it strongly enhances the correlated relaxivities.

In particular, the $r_{2}$ (the crucial parameter for a superparamagnetic iron oxide material) is 2 or 3 times higher than the corresponding value of the commercial contrast agent Endorem ${ }^{\circledR}$ at the typical frequencies of the clinical instrumentation [ca. 8.5, 21.3 and 63.9 MHz (magnetic fields of 0.2, 0.5 and $1.5 \mathrm{~T}$, respectively)]. Furthermore SPION@DMSA possess better $r_{1}$ values (more than one order of magnitude)

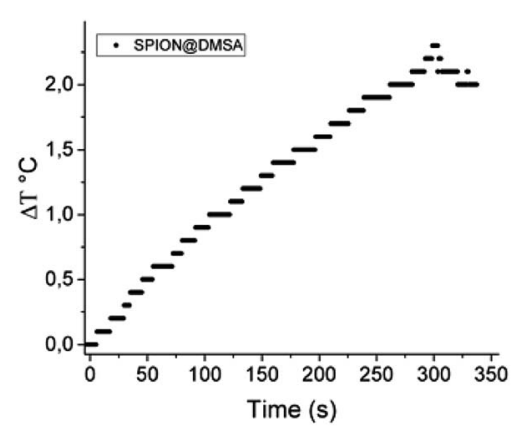

Fig. 7 Temperature kinetics of a water suspension of SPION@DMSA, $0.77 \mathrm{mg} \mathrm{mL}^{-1} \mathrm{Fe}$, during the exposure to the alternating magnetic field $\left(17.1 \mathrm{kA} \mathrm{m}^{-1}, 183 \mathrm{kHz}\right)$. The starting temperature was $295 \mathrm{~K}$. with respect to the routinely used positive contrast agent Dotarem ${ }^{\circledR}$ (see inset in Fig. 8a) at those frequencies/fields. This property opens the possibility to use SPION@DMSA as positive and negative contrast agent at the same time, depending on the experimental parameters used in the pulse NMR sequences during the acquisition of $\mathrm{MR}$ images. $^{35}$ Interestingly, SPION@DMSA shows no difference between $r_{1}$ and $r_{2}$ at the zero field limit (see inset in Fig. 8b) as predicted by theory, an experimental result obtained only recently in the literature. ${ }^{36}$ The equivalence of $r_{1}$ and $r_{2}$ at low proton Larmor frequencies would have been unattainable if our sample was not well dispersed in water without any unwanted particle aggregation.

The slight influence of the functionalization with PNA on the relaxivity values is explained by two characteristics of SPION@DMSA-Mal-PNA: on one side the higher steric hindrance as evinced by AFM (NP overall size $=25.6 \pm 2.6 \mathrm{~nm}$ (averaged value over 38 observations)), and on the other side the poor solubility of PNA sequence. Both these properties oblige the water molecules to stay slightly further from the magnetic core of the SPIONs or, at least, to follow a more difficult pathway
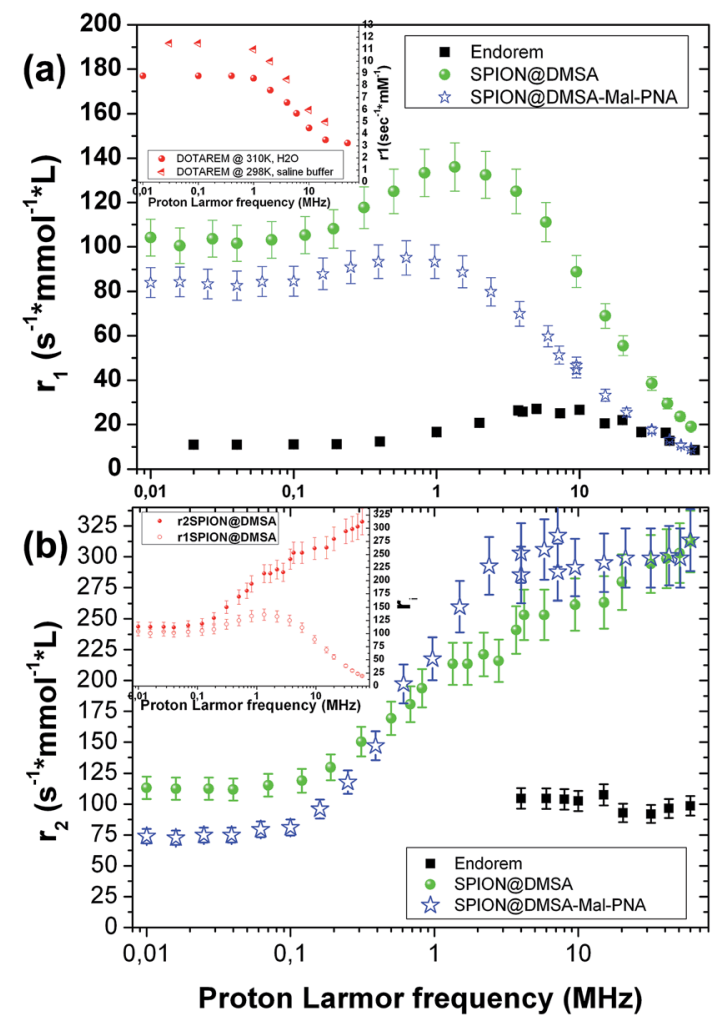

Fig. 8 Longitudinal (a) and transverse (b) NMRD profiles of SPION@DMSA (green circles) and SPIONaDMSA-Mal-PNA (blue stars) compared to commercial compound Endorem ${ }^{\circledR}$ (black squares). In the inset of panel $a$, the longitudinal NMRD profiles of the commercial contrast agent Dotarem dispersed in water or in saline buffer measured at two different temperatures are reported. The inset shows the extremely good performances of both our samples also as $T_{1}$ relaxing agent. In the inset of the panel $b$, longitudinal and transverse relaxivities of SPION@DMSA are plotted together (the same results are obtained also for SPIONQDMSA-Mal-PNA), to show the equivalence of $r_{1}$ and $r_{2}$ values at low frequency, as predicted by the theory (see the text). 
if compared to the case of SPION@DMSA. Consequently, mildly lower values for the $r_{1}$ NMR dispersion profile and for low proton Larmor frequencies of $r_{2}$ are found, while substantially the same $r_{2}$ values of SPION@DMSA sample are obtained at $\nu>$ $0.1 \mathrm{MHz}$.

Therefore, the PNA presence did not influence significantly the relaxivity efficiencies of these nanosystems, whereas the functionalization provides a significant added value.

\section{Conclusions}

A novel and effective strategy for binding PNA oligomers onto the surface of superparamagnetic iron oxide nanoparticles has been developed. All the requirements that stimulated this work have been fulfilled, and some of the drawbacks shown by previous literature reports have been overcome. The control of the size of the magnetic core for the diagnostic and therapeutic applications is ensured by the use of thermal decomposition synthetic route. The desired water solubility and the colloidal stability are provided by the coating with DMSA. The stable linking of SPION to PNA oligomers is given by the efficient Michael addition of maleimide-functionalized PNAs to the $\mathrm{SH}$ groups of DMSA grafted to the nanoparticle surface.

A detailed characterization of the morphological, magnetic and relaxivity properties of the SPION, which have been further conjugated to PNA, showed that these nanoparticles are wellsuited to increase both the positive and negative contrast of magnetic resonance imaging and to rise the temperature of their surroundings when subjected to an alternating magnetic field (with a SAR as high as $65 \mathrm{~W} \mathrm{~g}^{-1}$ ). Noteworthy, the conjugation of PNA to NPs did not affect their magnetic and relaxivity properties.

The approach here described, therefore, produces multimodal hybrid organic-inorganic nanomaterials, that can act as dual ( $T_{1}$ and $T_{2}$ ) MRI contrast agents, as efficient hyperthermia promoters and as PNA carriers. To fully exploit the theranostic potential of these nanohybrids, future work will explore the replacement of the standard PNA sequence, here used for setting the method, with PNA oligomers designed for targeting specific non-coding microRNAs (miRNA), ${ }^{37}$ whose dysregulation has been implicated in a variety of pathologies, such as inflammatory and autoimmune diseases, neurological disorders, as well as several types of cancer. ${ }^{38}$

\section{Experimental part}

\subsection{Materials}

All the reagents have been purchased by Sigma Aldrich (reagent grade) and used without further purification, if not otherwise specified. The PNA decamers were synthesized with standard automated Boc-based chemistry using commercially available monomers purchased from ASM Research Chemicals (Burgwedel, Germany). Polystyrene bead carrying 4-methylbenzhydrylamine hydrochloride salt groups (MBHA resin, $0.63 \mathrm{mmol} \mathrm{g}^{-1}$ ) was purchased from VWR International, and it was downloaded to $0.2 \mathrm{mmol} \mathrm{g}^{-1}$ with the thymine monomer Boc-PNA-T-OH as described elsewhere. ${ }^{25}$

\subsection{Instruments and methods}

Automated solid phase synthesis of resin-supported PNA 3 was performed with peptide synthesizer ABI 433A of Applera Italia, according to Applied Biosystems ABI 433A Peptide Synthesis 3 $\mathrm{mL}$ Reaction Vessel User's Manual for the MBHA resin. The software for peptide synthesis was Synassist 2.0.

Mass spectra were recorded on a ESI-Q-Tof Micro-Waters (Waters Corporation, Milford, MA), in the data-dependent acquisition and positive ion mode.

The reverse-phase RP-HPLC analyses were performed on Agilent 1100 series system, equipped with DAD analyser. The PNA purification was performed by RP-HPLC with UV detection at $\lambda 260 \mathrm{~nm}$ using the semi-preparative column Luna C18 $(25 \mathrm{~cm}$ $\times 10 \mathrm{~mm}, 5 \mu \mathrm{m}$ ), eluting with $\mathrm{H}_{2} \mathrm{O}$ containing $0.1 \%$ TFA (eluent A) and $\mathrm{CH}_{3} \mathrm{CN}$ containing $0.1 \%$ TFA (eluent B); elution gradient:

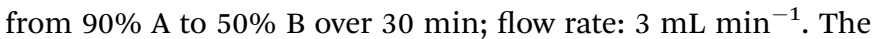
purity of PNA was evaluated by RP-HPLC using analytical column Luna C18 $(25 \mathrm{~cm} \times 4.6 \mathrm{~mm}, 5 \mu \mathrm{m})$, eluting with $\mathrm{H}_{2} \mathrm{O}$ containing $0.1 \%$ TFA (eluent A) and $\mathrm{CH}_{3} \mathrm{CN}$ containing $0.1 \%$ TFA (eluent B); elution gradient: from $95 \%$ A to $100 \%$ B over $60 \mathrm{~min}$; flow rate: $1 \mathrm{~mL} \mathrm{~min}^{-1}$.

High resolution NMR experiments were performed on a Bruker Model DRX400 spectrometer, equipped with a Bruker 5 $\mathrm{mm}$ BBI Z-gradient probe head capable of producing gradients with a strength of $53.5 \mathrm{G} \mathrm{cm}^{-1}$.

Infrared (IR) spectra were acquired on a Perkin-Elmer Spectrum BX FT-IR instrument. Samples were dispersed in $\mathrm{KBr}$ and pressed in a pellet. The IR spectra were registered between 4000 and $400 \mathrm{~cm}^{-1}$.

Electronic absorption spectra were recorded on an Agilent Model 8543 spectrophotometer at room temperature and using quartz cells with $1.0 \mathrm{~cm}$ path length.

Metal content on SPION was determined by AAS analysis on a Perkin-Elmer Pinaacle 900 instrument and for SPION7@DMSA was further confirmed by a spectrophotometric method. For AAS analysis few microliters of particle suspension were digested with $1 \mathrm{~mL}$ aqua regia/HCl overnight at RT in a $10 \mathrm{~mL}$ volumetric flask, and subsequently filled up with milliQ water. For spectrophotometric analysis few microliters of particle suspension were digested with $1 \mathrm{~mL}$ aqua regia/ $\mathrm{HCl}$ at $\mathrm{RT}$ in a $10 \mathrm{~mL}$ volumetric flask and then filled up with (i) $\mathrm{NH}_{2} \mathrm{OH}$ solution $(10 \% \mathrm{w} /$ w, $0.1 \mathrm{~mL}$ ), (ii) acetate buffer solution ( $\mathrm{pH} 4.6,0.15 \mathrm{M}, 6 \mathrm{~mL}$ ), (iii) 1,10-phenantroline solution $\left(0.6 \% \mathrm{w} / \mathrm{w}, \mathrm{H}_{2} \mathrm{O} / \mathrm{MeOH} 10: 1,0.2\right.$ $\mathrm{mL}$ ) (iv) milliQ water. The absorbance at $510 \mathrm{~nm}$ was then measured with an UV-Vis spectrophotometer and plotted against standards prepared with the same procedure starting from a commercial AAS standard solution.

Elemental $\mathrm{C}, \mathrm{H}, \mathrm{N}$ analyses were performed on a Perkin Elmer CHN 2400 instrument.

DLS and $\zeta$-potential measurements were carried out on a Zetasizer Nano ZS instrument (Malvern Instruments Corp., Malvern, Worcestershire, UK) at a wavelength of $633 \mathrm{~nm}$ with a solid state $\mathrm{He}-\mathrm{Ne}$ laser at a scattering angle of $173^{\circ}$, at $298 \mathrm{~K}$ on diluted samples (0.01-0.1 $\mathrm{mg} \mathrm{mL}^{-1}$ nanoparticles) at $\mathrm{pH} 7$. Each hydrodynamic diameter as well as $\zeta$-potential were averaged from at least three measurements. 
Average diameter and size distribution of the SPION were determined from TEM images recorded using a CM12 PHILIPS transmission electron microscope operating at $100 \mathrm{kV}$. Samples were prepared by drop drying a dilute solution of the samples onto 200 mesh carbon-coated copper grids. The recorded micrographs were further analyzed with the FIJI ${ }^{\circledR}$ software. The mean diameter and size distribution of each sample were obtained from a statistical analysis over 400 SPION.

The NMR-dispersion profiles were measured on water suspensions of each sample. Longitudinal and transverse nuclear relaxation times, $T_{1}$ and $T_{2}$, respectively, were evaluated over the range $10 \mathrm{kHz}$ to $60 \mathrm{MHz}$ for the ${ }^{1} \mathrm{H}$ (proton) Larmor frequency, corresponding to an applied magnetic field in the range $2.3 \times 10^{-4}$ to $1.41 \mathrm{~T}$. In order to cover such a wide range, two different instruments were used: a Stelar Spinmaster with a standard electromagnet for the range: 10-60 MHz; and a Stelar SMARtracer, working with the Fast-Field Cycling technology, for the low and very low fields' range, corresponding to $10 \mathrm{kHz}$ to 10 MHz. In the first case, standard pulse sequences were selected, that is, saturation recovery for $T_{1}$ and Car-Purcell-MeiboomGill (CPMG) for $T_{2}$. For the very low field range $(v<3.7 \mathrm{MHz}$ for $T_{1}$ and $v<4: 2 \mathrm{MHz}$ for $T_{2}$ ), ad hoc pre-polarized sequences were used to increase the NMR proton signal.

AFM imaging was performed using a Nanoscope Multimode IIId AFM (Bruker, Santa Barbara, CA, USA). Tapping-mode AFM images were collected in air using the root mean square amplitude of the cantilever as the feedback signal for the vertical sample position. The mica support (Ted Pella, CA, USA) was glued to a metal disk that was magnetically fixed to the AFM sample holder. Rectangular silicon nitride probes with nominal spring constant around $2.5 \mathrm{~N} \mathrm{~m}^{-1}$ (NSG01, NT-MDT, Russia) and cantilever length of $120 \mu \mathrm{m}$ were used for the tapping-mode imaging. The cantilever resonance frequency was about $130 \mathrm{kHz}$. The root mean square free amplitude of the cantilever was approximately $15 \mathrm{~nm}$ and the relative set-point above $95 \%$ of the free amplitude. Images were recorded at $\sim 1 \mathrm{~Hz}$ line rate, and a resolution of 512 $\times 512$ pixels per image was chosen. AFM images were subjected to a line-by-line subtraction of linear background ("flattening"), to eliminate sample tilt from the images and correct for stepwise changes between individual scan lines by using the Nano-Scope III software (version S.31R1, Bruker, USA).

Magnetic measurements were performed using a Quantum Design MPMS SQUID magnetometer operating in the $1.8-350 \mathrm{~K}$ temperature range and with an applied field up to $50 \mathrm{kOe}$. Measurements were performed on pressed powder of SPION sample. All data were corrected for the diamagnetic contribution of the sample holder, and were normalized to the amount of magnetic material as evaluated from elemental analysis. Zero Field Cooled-Field Cooled (ZFC/FC) curves were obtained by measuring the temperature dependence of the magnetization applying a probe magnetic field (50 Oe), after cooling the sample in the absence (ZFC) or in the presence (FC) of the field. A rough estimate of $T_{\mathrm{B}}$ value accuracy is given by the half interval between the temperatures of two subsequent acquisition points, i.e., $2.5 \mathrm{~K}$. The field dependence of the magnetic moment ( $M v s . H$ ) was measured cycling the field between $\pm 50 \mathrm{kOe}$ at $2.5 \mathrm{~K}$ and $300 \mathrm{~K}$. The accuracy of $H_{\mathrm{C}}, M_{\mathrm{R}}$ and $M_{\mathrm{S}}$ evaluation can be estimated as low as $3 \%$.
The determination of Specific Absorption Rate (SAR) was performed through calorimetric measurements by recording temperature kinetics of SPION suspension exposed to an alternating magnetic field. Measurements were performed adapting a commercial setup, composed by a $6 \mathrm{~kW}$ Fives Celes power supply, a water-cooled induction coil and a series of variable capacitors ( $420 \mathrm{nF}$ to $4.8 \mu \mathrm{F}$ ) for setting the required frequency. Such resonant RLC circuit is able to produce an alternating magnetic field in the range of $50-400 \mathrm{kHz}$ and with amplitude up to $19.1 \mathrm{kA} \mathrm{m}^{-1}$. The frequency and field amplitude values used in this work $\left(183 \mathrm{kHz}, 17.0 \mathrm{kA} \mathrm{m}^{-1}\right)$ were chosen in order to operate under the physiological limit, $H \nu<5 \times 10^{9} \mathrm{~A} \mathrm{~m}^{-1} \mathrm{~s}^{-1}$, beyond which deleterious responses of living tissues are observed. The sample was placed in the middle of the induction coil, inside a polystyrene sample holder placed in a glass Dewar connected to a glycol thermal bath in order to isolate the sample bath from the thermal gradient of the coils and from the environment. The real amplitude of the magnetic field was determined by a AMF Life Systems high frequency probe. Measurements of the sample temperature were performed by an optical fiber thermometer connected to a digital temperature recorder (Fotemp). The SAR values were calculated using the equation $\mathrm{SAR}=\left(\Sigma_{\mathrm{i}} m_{\mathrm{i}} c_{\mathrm{i}} / m_{\mathrm{me}}\right)(\Delta T /$ $\Delta t$ ), where $\Delta T$ is the temperature increase in the interval of time $\Delta t, m_{\mathrm{me}}$ is the total mass of metal, $m_{\mathrm{i}}$ is the mass in grams of the $\mathrm{i}$ species and $c_{\mathrm{i}}$ is its specific heat. The sum is extended to all the $\mathrm{i}$ species involved in the heat exchange. Since the measurements are carried in non adiabatic conditions, the $\Delta T / \Delta t$ values were extrapolated for $t \approx 0$ from temperature kinetic curves, by considering the initial slope.

\subsection{Synthesis of SPION7@OA}

The synthesis of SPION was performed following a slightly modified literature procedure. ${ }^{\mathbf{1 1}}$ Briefly, in a $50 \mathrm{~mL}$ three-necked round bottom flask $13.9 \mathrm{mmol}$ of oleic acid $(4.38 \mathrm{~mL}, d=$ $0.895 \mathrm{~g} \mathrm{~mL}^{-1} ; 70 \%$ purity) and $2 \mathrm{mmol}$ of $\mathrm{Fe}(\mathrm{CO})_{5}(236 \mu \mathrm{L}, d=$ $1.49 \mathrm{~g} \mathrm{~mL}^{-1}$ ) were dissolved under inert atmosphere in $4 \mathrm{~mL}$ of 1-octadecene. This solution was then heated from room to $320^{\circ} \mathrm{C}$ at a rate of $15^{\circ} \mathrm{C} \min ^{-1}$ and refluxed for $3 \mathrm{~h}$. During this time the reddish solution turned to black. The solution was then cooled to $120{ }^{\circ} \mathrm{C}$ at a rate of $5{ }^{\circ} \mathrm{C} \mathrm{min}{ }^{-1}$ and maintained at that temperature for 2 more hours in air to allow oxidation of $\mathrm{Fe}(0)$. The solution was cooled down to room temperature at a rate of $5{ }^{\circ} \mathrm{C} \mathrm{min}^{-1}$. The SPION were subsequently isolated by centrifugation of the reaction mixture after addition of $\mathrm{ca} .70$ $\mathrm{mL}$ of acetone $(3 \times$ at 7197 round centrifugal force, $\mathrm{rcf})$ followed by removal of the yellow supernatant. The isolated particles were then re-suspended in $15 \mathrm{~mL}$ of hexane and stored under nitrogen atmosphere at $-25{ }^{\circ} \mathrm{C}$ for further uses.

The same procedure was followed for the synthesis of SPION6@OA and SPION8@OA, using different amounts of oleic acid (1.90 $\mathrm{mL}$ and $4.9 \mathrm{~mL}$, respectively), corresponding to 6 and 8 equivalents, with respect to $\mathrm{Fe}(\mathrm{CO})_{5}$.

\subsection{SPION@DMSA}

A sample of SPION7@OA in hexane solution (3 mL, $11 \mathrm{mg} \mathrm{mL}^{-1}$ Fe as measured by AAS) was treated with $8 \mathrm{~mL}$ of acetone and 
centrifuged (4 min, $7197 \mathrm{rcf}$ ) to remove the excess oleic acid. The nanoparticles were then suspended in $9 \mathrm{~mL}$ of hexane and moved to a Schlenk flask under nitrogen atmosphere. To this suspension a solution of DMSA dissolved in acetone $(43.5 \mathrm{mg}$ in $9 \mathrm{~mL}$ ) was added, followed by $15 \mu \mathrm{L}$ of TEA. The Schlenk flask was then moved to an ultrasonic bath and the reaction mixture was kept at $50{ }^{\circ} \mathrm{C}$ for $40 \mathrm{~min}$, maintaining the suspension under nitrogen atmosphere for the whole sonication time. The SPION were then collected with a magnet and then centrifuged first with $20 \mathrm{~mL}$ of acetone (10 min, $7197 \mathrm{rcf}$ ) and then with $20 \mathrm{~mL}$ of water (30 $\mathrm{min}, 7197 \mathrm{rcf}$ ). The washed SPION were then resuspended in $10 \mathrm{~mL}$ of water and stored under nitrogen atmosphere at $4{ }^{\circ} \mathrm{C}$ for further uses.

\subsection{Preparation of the resin-supported PNA decamer 3}

Automated solid phase synthesis was performed on an ABI 433A peptide synthesizer in a reactor of $3 \mathrm{~mL}$ on a $20 \mu \mathrm{M}$ scale using Boc strategy. The MBHA resin downloaded with thymine monomer (100 mg, $0.2 \mathrm{mmol} \mathrm{g}^{-1}$ ) was swollen with $\mathrm{CH}_{2} \mathrm{Cl}_{2}$, the Boc group of the loaded monomer was removed by treatment with TFA/m-cresol (95:5), the resin was rinsed with $\mathrm{CH}_{2} \mathrm{Cl}_{2}$ and DIPEA 1.6 $\mathrm{M}$ in NMP. The monomers were loaded into cartridges as NMP solutions, activated with HBTU $0.38 \mathrm{M}$ in NMP and transferred to the resin. After each coupling step (30 min) the resin was washed with NMP and then treated with $\mathrm{Ac}_{2} \mathrm{O} / \mathrm{Py} / \mathrm{NMP} 1: 25: 25$ capping solution twice for $3 \mathrm{~min}$. The cycle was repeated for each base. After the last coupling the resin was washed several times with NMP and $\mathrm{CH}_{2} \mathrm{Cl}_{2}$, and finally dried under nitrogen yielding 3 (128 mg).

\subsection{Synthesis of PNA decamer 1}

$50 \mathrm{mg}$ of resin was washed with TFA $(2 \times 200 \mu \mathrm{L})$ and then stirred for $1 \mathrm{~h}$ with a solution of TFA/TFMSA/thioanisole $/ m$ cresol $6: 2: 1: 1(500 \mu \mathrm{L})$. The mixture was filtered and the resin washed with TFA $(4 \times 200 \mu \mathrm{L})$. The filtrate was concentrated, and $\mathrm{Et}_{2} \mathrm{O}(5 \mathrm{~mL})$ was added to precipitate PNA as a white solid. Centrifugation of the slurry gave the product, which was washed with $\mathrm{Et}_{2} \mathrm{O}(3 \times 5 \mathrm{~mL})$, and dried. The crude product was purified by RP-HPLC to afford the decamer $\mathbf{1}$ as a colourless solid (15 mg). Analytical RP-HPLC: $t_{\mathrm{R}}=8.86 \mathrm{~min}$. ESI MS: $\mathrm{m} / z$ found 1363.9 $\left[\mathrm{MH}_{2}\right]^{+}, 909.6\left[\mathrm{MH}_{3}\right]^{+}, 682.4\left[\mathrm{MH}_{4}\right]^{+}, 546.2\left[\mathrm{MH}_{5}\right]^{+}$; $M_{\mathrm{r}}$ calcd: 2726.6 .

\subsection{Synthesis of PNA decamer 2}

The resin 3 ( $50 \mathrm{mg}, 0.2 \mathrm{mmol} \mathrm{g}{ }^{-1}$ ) was swollen with $\mathrm{CH}_{2} \mathrm{Cl}_{2}$ (3 $\mathrm{mL}$ ) for $1 \mathrm{~h}$, then the Boc group of the last monomer of the sequence was removed by treatment with a solution of TFA $/ m$ cresol (95:5). The resin was rinsed with $\mathrm{CH}_{2} \mathrm{Cl}_{2}$ and NMP. In a vial, a solution of DIPEA (17 $\mu \mathrm{L}, 0.1 \mathrm{mmol}, 10 \mathrm{eq}$.) and the $p$ maleimidobenzoic acid (11.9 mg, $0.055 \mathrm{mmol}, 5.5$ eq.) in NMP $(0.4 \mathrm{~mL})$ was added to a solution of HATU $(19.0 \mathrm{mg}, 0.05 \mathrm{mmol}$, 5 eq.) in NMP, and the resulting mixture was shaken for two minutes. The activated mixture was then added to the resin and shaken for $2 \mathrm{~h}$. The resin was then washed with TFA $(2 \times 200$ $\mu \mathrm{L}$ ), and subsequently stirred for $1 \mathrm{~h}$ with a solution of TFA/ TFMSA/thioanisole/m-cresol $6: 2: 1: 1(500 \mu \mathrm{L})$. The mixture was filtered, and the resin washed with TFA $(4 \times 200 \mu \mathrm{L})$. The filtrate was concentrated, and $\mathrm{Et}_{2} \mathrm{O}$ was added to precipitate PNA as a white solid. Centrifugation of the slurry gave the product, which was washed with $\mathrm{Et}_{2} \mathrm{O}(3 \times 5 \mathrm{~mL})$, and dried. The crude PNA was purified by RP-HPLC to afford the PNA 2 (11 $\mathrm{mg}$ ) as a white solid. Analytical RP-HPLC: $t_{\mathrm{R}}=10.2 \mathrm{~min}$. ESI MS: $m / z$ found $1463.6\left[\mathrm{MH}_{2}\right]^{+}, 976.1\left[\mathrm{MH}_{3}\right]^{+}, 732.3\left[\mathrm{MH}_{4}\right]^{+}, 585.8$ $\left[\mathrm{MH}_{5}\right]^{+} ; M_{\mathrm{r}}$ calcd: 2925.3.

\subsection{Preparation of SPION@DMSA-PNA}

The SPION@DMSA carboxyl groups were first activated by adding $12.4 \mathrm{mg}$ EDC (0.065 mmol) and $46 \mathrm{mg}$ NHS $(0.4 \mathrm{mmol})$ to $5 \mathrm{~mL}$ of SPION@DMSA water suspension $\left(0.56 \mathrm{mg} \mathrm{mL}^{-1} \mathrm{Fe}\right)$. The addition of EDC apparently destabilized the nanoparticle colloid, resulting in visible aggregation. The suspension $\mathrm{pH}$ was then changed to 4.5 by careful addition of $\mathrm{HCl}$ and the activation reaction continued for $2 \mathrm{~h}$ in an ultrasonic bath. Then the activated SPION were added dropwise to a solution of $5 \mathrm{mg}$ PNA 1 dissolved in $0.5 \mathrm{~mL}$ of $\mathrm{CH}_{3} \mathrm{CN}$ and $1 \mathrm{~mL}$ of phosphate buffer ( $\mathrm{pH} 8,5 \mathrm{mM})$. The suspension $\mathrm{pH}$ was adjusted to 8 by careful addition of $\mathrm{NaOH}$ solution. The reaction mixture was first placed in an ultrasonic bath and left under ultrasonic irradiation for $2 \mathrm{~h}$ at room temperature and then left on a shaking plate overnight. The nanoparticles were then purified via centrifugation with water $(3 \times, 15 \mathrm{~min}, 7197 \mathrm{rcf})$ and subsequently collected in a Schlenk flask under inert atmosphere.

\subsection{Ellman's essay}

An aliquot of $200 \mu \mathrm{L}$ of SPION@DMSA suspension $(0.56 \mathrm{mg}$ $\mathrm{mL}^{-1} \mathrm{Fe}$ ) was diluted with $3 \mathrm{~mL}$ of phosphate buffer $(\mathrm{pH} 8.3$, $0.05 \mathrm{M}$ ). To this suspension $1 \mathrm{~mL}$ of Ellman's reagent solution (3 $\mathrm{mg}$ dissolved in $10 \mathrm{~mL}$ phosphate buffer) was added. As a comparison two solutions, containing only SPIONs and only Ellman's reagent, respectively, were prepared. After $90 \mathrm{~min}$ of incubation in the dark the suspensions were centrifuged and the supernatant absorbance at $412 \mathrm{~nm}$ was measured by UV-Vis spectroscopy (Fig. S8, ESI $\dagger$ ).

\subsection{Synthesis of the rhodamine B-maleimide adduct}

The synthesis (depicted in Scheme S1, ESI $\dagger$ ) involved at first the preparation of mono-protected $\mathrm{N}$-Boc-ethylenediamine, that was performed according to a literature procedure. ${ }^{39} \mathrm{~N}$-(2-Aminoethyl)maleimide was then prepared by another literature method,,$^{\mathbf{4}}$ and reacted with rhodamine B isothiocyanate, in an NMR tube under nitrogen atmosphere: $3.3 \mathrm{mg}$ of rhodamine BNCS $\left(6.15 \times 10^{-3} \mathrm{mmol}\right)$ were dissolved in $0.5 \mathrm{~mL}$ of deuterated methanol and treated with $1.6 \mathrm{mg}$ of $\mathrm{N}$-(2-aminoethyl)maleimide trifluoroacetate salt $\left(5.12 \times 10^{-3} \mathrm{mmol}\right)$ and $0.85 \mu \mathrm{L}$ of $\mathrm{Et}_{3} \mathrm{~N}$. The reaction was carried out overnight at RT and its progress was monitored following the decrease of the sharp singlet at $6.91 \mathrm{ppm}$ due to the $\mathrm{CH}$ on double bond of $\mathrm{N}-(2$ aminoethyl)maleimide that, once reacted, slightly shifted to upper fields (ca. $6.89 \mathrm{ppm}$ ) and split in multiple singlets due to the different isomers. The concentration of rhodamine-Bmaleimide adduct was estimated to be $0.006 \mathrm{M}$ using its known molar attenuation coefficient $(\varepsilon=87000$ at $559 \mathrm{~nm}){ }^{41}$ 


\subsection{Reaction between rhodamine B-maleimide adduct and} SPION@DMSA

In a Schlenk flask under nitrogen atmosphere $100 \mu \mathrm{L}$ of SPION@DMSA $\left(0.78 \mathrm{mg} \mathrm{mL}{ }^{-1} \mathrm{Fe}\right)$ were diluted with $2 \mathrm{~mL}$ milliQ water. To this suspension $17 \mu \mathrm{L}$ of the functionalized rhodamine solution were added. The reaction was incubated in the dark for $24 \mathrm{~h}$. The nanoparticles were collected by centrifugation $(20 \mathrm{~min} \times 7197 \mathrm{rcf}$ ) and re-dispersed in water for three times, in order to remove all the unreacted Rhodamine-B. The purified nanoparticles were then suspended in $2 \mathrm{~mL}$ milliQ water and their absorbance was measured via UV-Vis spectroscopy (Fig. S6, ESI $\dagger$ ).

\subsection{Preparation of SPION@DMSA-Mal-PNA}

A suspension of $5 \mathrm{~mL}$ of SPION@DMSA $\left(0.56 \mathrm{mg} \mathrm{mL}^{-1} \mathrm{Fe}\right)$ was added to a solution of $5 \mathrm{mg}$ of PNA 2 dissolved in $0.5 \mathrm{~mL}$ of milliQ water under inert atmosphere. The $\mathrm{pH}$ of the reaction mixture was adjusted to 7 and the reaction carried out under ultrasonic irradiation for $4 \mathrm{~h}$ at room temperature. The nanoparticles were then collected with a permanent magnet, resuspended in $5 \mathrm{~mL}$ water and centrifuged $(3 \times, 15 \mathrm{~min}$ at 7197 rcf). The isolated SPION were then stored under nitrogen atmosphere.

\section{Acknowledgements}

The authors thank CARIPLO Foundation for having supported this research (project no. 2013-0752, project title "New biomimetic tools for miRNAtargeting").

\section{Notes and references}

1 (a) J. Huang, Y. Li, A. Orza, Q. Lu, P. Guo, L. Wang, L. Yang and H. Mao, Adv. Funct. Mater., 2016, 26, 3818-3836; (b) C. Blanco-Andujar, A. Walter, G. Cotin, C. Bordeianu, D. Mertz, D. Felder-Flesch and S. Begin-Colin, Nanomedicine, 2016, 11, 1889-1910; (c) H. Liu, J. Zhang, X. Chen, X.-S. Du, J.-L. Zhang, G. Liu and W.-G. Zhang, Nanoscale, 2016, 8, 7808-7826; (d) Y. Piñeiro, Z. Vargas, J. Rivas and M. A. Lõpez-Quintela, Eur. J. Inorg. Chem., 2015, 27, 4495-4509; (e) D. Ling, N. Lee and T. Hyeon, Acc. Chem. Res., 2015, 48, 1276-1285; ( $f$ ) L. Zhang, W. Dong and H. Sun, Nanoscale, 2013, 5, 7664-7684; $(g)$ E. Amstad, M. Textor and E. Reimhult, Nanoscale, 2011, 3, 2819-2843; (h) A. Figuerola, R. Di Corato, L. Manna and T. Pellegrino, Pharmacol. Res., 2010, 62, 126-143; (i) M. Mahmoudi, V. Serpooshan and S. Laurent, Nanoscale, 2011, 3, 30073026; (j) A. G. Roca, R. Costo, A. F. Rebolledo, S. Veintemillas-Verdaguer, P. Tartaj, T. González-Carreño, M. P. Morales and C. J. Serna, J. Phys. D: Appl. Phys., 2009, 42, 224002; (k) C. C. Berry, J. Phys. D: Appl. Phys., 2009, 42, 224003; ( $l$ ) Q. A. Pankhurst, N. T. K. Thanh, S. K. Jones and J. Dobson, J. Phys. D: Appl. Phys., 2009, 42, 224001; (m) J. Gao, H. Gu and B. Xu, Acc. Chem. Res., 2009, 42, 10971107; (n) S. Laurent, D. Forge, M. Port, A. Roch, C. Robic,
L. Vander Elst and R. N. Muller, Chem. Rev., 2008, 108, 2064-2110.

2 (a) P. Arosio, J. Thévenot, T. Orlando, F. Orsini, M. Corti, M. Mariani, L. Bordonali, C. Innocenti, C. Sangregorio, H. Oliveira, S. Lecommandoux, A. Lascialfari and O. Sandre, J. Mater. Chem. B, 2013, 1, 5317-5328; (b) V. Amendola, M. Meneghetti, O. M. Bakr, P. Riello, S. Polizzi, D. H. Anjum, S. Fiameni, P. Arosio, T. Orlando, C. D.-J. Fernandez, F. Pineider, C. Sangregorio and A. Lascialfari, Nanoscale, 2013, 5, 5611-5619; (c) N. Lee, Y. Choi, Y. Lee, M. Park, W. K. Moon, S. H. Choi and T. Hyeon, Nano Lett., 2012, 12, 3127-3131; (d) E. Poselt, H. Kloust, U. Tromsdorf, M. Janschel, C. Hahn, C. Maßlo and H. Weller, ACS Nano, 2012, 6, 1619-1624; (e) L. Xiao, J. Li, D. F. Brougham, E. K. Fox, N. Feliu, A. Bushmelev, A. Schmidt, N. Mertens, F. Kiessling, M. Valldor, B. Fadeel and S. Mathur, ACS Nano, 2011, 5, 6315-6324; (f) M. F. Casula, P. Floris, C. Innocenti, A. Lascialfari, M. Marinone, M. Corti, R. A. Sperling, W. J. Parak and C. Sangregorio, Chem. Mater., 2010, 22, 1739-1748.

3 Q. A. Pankhurst, J. Connolly, S. K. Jones and J. Dobson, J. Phys. D: Appl. Phys., 2003, 36, R167-R181.

4 (a) G. F. Goya, V. Grazu and M. R. Ibarra, Curr. Nanosci., 2008, 4, 1-16; (b) K. Maier-Hauff, R. Rothe, R. Scholz, U. Gneveckow, P. Wust, B. Thiesen, A. Feussner, A. von Deimling, N. Waldoefner, R. Felix and A. Jordan, J. NeuroOncol., 2007, 81, 53-60; (c) M. Mallory, E. Gogineni, G. C. Jones, L. Greer and C. B. Simone, Crit. Rev. Oncol. Hematol., 2016, 97, 56-64.

5 (a) A. Bhirde, J. Xie, M. Swierczewska and X. Chen, Nanoscale, 2011, 3, 142-153; (b) M. Mahmoudi, H. Hosseinkhani, M. Hosseinkhani, S. Boutry, A. Simchi, W. S. Journeay, K. Subramani and S. Laurent, Chem. Rev., 2011, 111, 253280.

6 (a) Z. P. Xu, Q. H. Zeng, G. Q. Lu and A. B. Yu, Chem. Eng. Sci., 2006, 61, 1027-1040; (b) J. Dobson, Gene Ther., 2006, 13, 283287.

7 (a) P. E. Nielsen, M. Egholm, R. H. Berg and O. Buchardt, Science, 1991, 254, 1497-1500; (b) P. Wittung, P. E. Nielsen, O. Buchardt, M. Egholm and B. Norden, Nature, 1994, 368, 561-563.

8 G. Prencipe, S. Maiorana, P. Verderio, M. Colombo, P. Fermo, E. Caneva, D. Prosperi and E. Licandro, Chem. Commun., 2009, 40, 6017-6019.

9 S. Khadsai, B. Rutnakornpituk, T. Vilaivan, M. Nakkuntod and M. Rutnakornpituk, J. Nanopart. Res., 2016, 18, 263.

10 (a) S. Sun, H. Zeng, D. B. Robinson, S. Raoux, P. M. Rice, S. X. Wang and G. Li, J. Am. Chem. Soc., 2004, 126, 273279; (b) J. Park, K. An, Y. Hwang, J.-G. Park, H.-J. Noh, J.-Y. Kim, J.-H. Park, N.-M. Hwang and T. Hyeon, Nat. Mater., 2004, 3, 891-895; (c) F. X. Redl, C. T. Black, G. C. Papaefthymiou, R. L. Sandstrom, M. Yin, H. Zeng, C. B. Murray and S. P. O'Brien, J. Am. Chem. Soc., 2004, 126, 14583-14599.

11 P. Calcagnile, D. Fragouli, I. S. Bayer, G. C. Anyfantis, L. Martiradonna, P. D. Cozzoli, R. Cingolani and A. Athanassiou, ACS Nano, 2012, 6, 5413-5419. 
12 T. Hyeon, S. S. Lee, J. Park, Y. Chung and H. B. Na, J. Am. Chem. Soc., 2001, 123, 12798-12801.

13 (a) A. Lassenberger, O. Bixner, T. Gruenewald, H. Lichtenegger, R. Zirbs and E. Reimhult, Langmuir, 2016, 32, 4259-4269; (b) R. Hufschmid, H. Arami, R. M. Ferguson, M. Gonzales, E. Teeman, L. N. Brush, N. D. Browning and K. M. Krishnan, Nanoscale, 2015, 7, 11142-11154; (c) K. Woo, J. Hong, S. Choi, H.-W. Lee, J.-P. Ahn, C. S. Kim and S. W. Lee, Chem. Mater., 2004, 16, 2814-2818.

14 E. Sevinç, F. S. Ertas, G. Ulusoy, C. Ozen and H. Y. Acar, J. Mater. Chem., 2012, 22, 5137-5144.

15 A. Ruiz, P. C. Morais, R. Bentes de Azevedo, Z. G. M. Lacava, A. Villanueva and M. del Puerto Morales, J. Nanopart. Res., 2014, 16, 2589-2609.

16 (a) A. G. Roca, S. Veintemillas-Verdaguer, M. Port, C. Robic, C. J. Serna and M. P. Morales, J. Phys. Chem. B, 2009, 113, 7033-7039; (b) M. Song, Y. Zhang, S. Hu, L. Song, J. Dong, Z. Chen and N. Gu, Colloids Surf., A, 2012, 408, 114-121.

17 O. F. Odio, L. Lartundo-Rojas, P. Santiago-Jacinto, R. Martínez and E. Reguera, J. Phys. Chem. C, 2014, 118, 2776-2791.

18 In TM-AFM, the sample is scanned by an oscillating cantilever whose oscillation amplitude is sensitive to both topography and mechanical properties of the sample surface. A phase angle is associated with the cantilever oscillation. A shift on the phase angle results from differences on AFM tip-sample interactions in which viscoelasticity plays an important role.

19 S. Yoshite and Y. Yamada, Eur. J. Biochem., 1979, 101, 395-399.

20 G. T. Hermanson, Bioconjugate techniques, 2nd edn, Academic Press - Elsevier, 2008.

21 A. Ruiz, G. Salas, M. Calero, Y. Hernández, A. Villanueva, F. Herranz, S. Veintemillas-Verdaguer, E. Martínez, D. F. Barber and M. P. Morales, Acta Biomater., 2013, 9, 6421-6430.

22 F. Xiong, Z. Y. Zhu, C. Xiong, X. Q. Hua, X. H. Shan, Y. Zhang and N. Gu, Pharm. Res., 2012, 29, 1087-1097.

23 Y. Hou, Y. Liu, Z. Chen, N. Gu and J. Wang, J. Nanobiotechnol., 2010, 8, 25 (14pp).

24 L. Tang, T. M. Fan, L. B. Borst and J. Cheng, ACS Nano, 2012, 6, 3954-3966.

25 G. Prencipe, S. Maiorana, P. Verderio, M. Colombo, P. Fermo, E. Caneva, D. Prosperi and E. Licandro, Chem. Commun., 2009, 40, 6017-6019.

26 A. L. Andrade, D. M. Souza, M. C. Pereira, J. D. Fabris and R. Z. Domingues, Cerâmica, 2009, 55, 420-424.

27 K. Nakamoto, Infrared and Raman Spectra of Inorganic and Coordination Compounds. Part B: Applications in Coordination, Organometallic, and Bioinorganic Chemistry, John Wiley, 6th edn, 2009.

28 See S. Ghosh and R. Mukhopadhyay, J. Colloid Interface Sci., 2011, 360, 52-60, and refs therein.
29 Details on magnetic hyperthermia theory can be found for example in (a) R. E. Rosenweig, J. Magn. Magn. Mater., 2002, 252, 370-374; (b) L.-M. Lacroix, R. Bel Malaki, J. Carrey, S. Lachaize, G. F. Goya, B. Chaudret and M. Respaud, J. Appl. Phys., 2009, 105, 023911.

30 K. Maier-Hauff, F. Ulrich, D. Nestler, H. Niehoff, P. Wust, B. Thiesen, H. Orawa, V. Budach and A. Jordan, J. NeuroOncol., 2011, 103, 317-324.

31 A. Lascialfari, M. Filibian, C. Sangregorio and P. Carretta, $L a$ Rivista del Nuovo Cimento, 2013, 6, 211-271.

32 (a) R. C. O'Handley, Modern Magnetic Materials Principles and Application, Wiley, New York, 2000; (b) U. Jeong, X. Teng, Y. Wang, H. Yang and Y. Xia, Adv. Mater., 2007, 19, 33-60.

33 L. Lartigue, C. Innocenti, T. Kalaivani, A. Awwad, M. del Mar Sanchez, Y. Guari, J. Larinova, C. Guérin, J. L. G. Montero, V. Barragan-Montero, P. Arosio, A. Lascialfari, D. Gatteschi and C. Sangregorio, J. Am. Chem. Soc., 2011, 133, 1045910472.

34 L. Kopanja, I. Milosevic, M. Panjan, V. Damnjanovic and M. Tadic, Appl. Surf. Sci., 2016, 362, 380-386.

35 The signal intensity SI, and consequently the image contrast in the MRI, is proportional to $\operatorname{SI}(\mathrm{TR}, \mathrm{TE}) \rho\left(1-\mathrm{e}^{-\mathrm{TR} / \mathrm{T} 1}\right) \mathrm{e}^{-\mathrm{TE} /}$ T2, where $\rho$ is the density of tissue nuclear spins, TR the repetition time of the radio frequency (rf) pulse sequence (i.e. the time between consecutive identical sequences) and TE the echo time (i.e. the time between the initial rf pulse and the time at which the signal is received). As a consequence, using the appropriate pulse sequences, it is possible to weight the image on T1 or T2 taking full advantage of the SPION@DMSA property (in detail T1weighted sequences use short TR and short TE), while T2weighted sequences use long TR and long TE.

36 (a) Y. Gossuin, T. Orlando, M. Basini, D. Henrard, A. Lascialfari, C. Mattea, S. Stapf and Q. L. Vuong, Nanotechnology, 2016, 27, 155706 (11pp); (b) T. Orlando, M. Albino, F. Orsini, C. Innocenti, M. Basini, P. Arosio, C. Sangregorio, M. Corti and A. Lascialfari, J. Appl. Phys., 2016, 119, 134301.

37 See for instance: (a) L. He and G. J. Hannon, Nat. Rev. Genet., 2004, 5, 522-531; (b) J. Krol, I. Loedige and W. Filipowicz, Nat. Rev. Genet., 2010, 11, 597-610.

38 M. V. Iorio and C. M. Croce, EMBO Mol. Med., 2012, 4, 143159.

39 M. Pittelkow, R. Lewinsky and J. B. Christensen, Org. Synth., 2007, 84, 209-214.

40 M. Richter, A. Chakrabarti, I. R. Ruttekolk, B. Wiesner, M. Beyermann, R. Brock and J. Rademann, Chem.-Eur. J., 2012, 18, 16708-16715.

41 O. Ma, M. Lavertu, J. Sun, S. Nguyen, M. D. Buschmann, F. M. Winnik and C. D. Hoemann, Carbohydr. Polym., 2008, 72, 616-624. 